\title{
"VA-ET-VIENT" ("BACK-AND-FORTH”) CCD SPECTROSCOPY: A NEW WAY TO INCREASE THE LIMITING MAGNITUDE OF VERY LARGE TELESCOPES
}

\author{
G. Soucail, J. C. Cuillandre, \\ Observatoire Midi-Pyrénées, Toulouse
}

J. P. Picat and

Observatoire Midi-Pyrénées, Bagnères-de-Bigorre

B. Fort

DEMIRM, Observatoire de Paris

\section{INTRODUCTION}

Over the last decade, the quantity of scientific results brought by the observations of very faint objects has been quite spectacular. In particular, they concern the photometry of faint galaxies up to $\mathrm{B}=27$ (Tyson 1988) or $\mathrm{K}=22$ (Cowie et al. 1994). The consequences of these observations are the detection of a large population of faint galaxies more numerous than any prediction given by standard galaxy evolution and probably a new vision of the distant universe. For faint object spectroscopy, the most recent surveys of field galaxies reach a magnitude range of 23 - 24 (Colless et al. 1990, 1993, Lilly and Cowie 1993, Tresse et al. 1993) with a reasonable $\mathrm{S} / \mathrm{N}$ ratio that allows a redshift measurement from absorption-line identification. But in this magnitude range, the sky background flux is dominant with respect to the source, being at least 10 times brighter. It is well known that in that case, with a low readout noise detector and a "perfect" spectrograph, the $\mathrm{S} / \mathrm{N}$ ratio scales as:

where $\mathrm{D}$ is the telescope diameter, $\mathrm{T}$ is the exposure time and $\mathrm{R}$ the resolution of the spectrograph. In practice, the $\mathrm{S} / \mathrm{N}$ does not increase as expected and tends to saturate towards an upper limit depending on the characteristics of the instrument but not on the telescope size. The main limitations come from residuals in the flatfielding process or from a bad sampling of the strong sky emission lines resulting in a poor sky subtraction. As a figure, this limit is typically of a few hours on a four-meter telescope with a low resolution of $\mathrm{R}=300$. Extrapolating to the next generation of eight to ten-meter class telescopes, it is clear that these limitations will become crucial as people will require to reach fainter magnitudes. Moreover, even now, faint object spectroscopy at very low resolution (such as $R=100$ ) is hardly possible despite the scientific interests, so what will happen with the VLTs? 
In this framework, we examine more quantitatively the main limitations which can occur in faint objects spectroscopy and propose a new mode of observations which we call "Va-etVient" ("Back-and-forth", Cuillandre et al. 1994). It is based on several shifts of the charges on the CCD related to a shift of the telescope from the object to an empty region of the sky, the detector being read only once at the end of the total exposure. We have tested this method in a laboratory experiment and proved its advantages over standard long slit spectroscopy in some specific cases. The implementation of the "Va-et-Vient" on a telescope is rather simple and is briefly described at the end of the paper as well as some future investigations of the method.

\section{QUANTITATIVE ESTIMATES OF THE S/N RATIO AND ITS LIMITATIONS}

To understand the main limitations which occur when observing faint objects with a spectrograph, let us write formally the $\mathrm{S} / \mathrm{N}$ ratio of the observed data. We call $\Phi_{\mathrm{O}}$ the true object signal per second recorded through a spectral element of the instrument and $\Phi_{\mathrm{S}}$ the sky signal. Both of them can be written as:

$$
\Phi \propto \frac{D^{2} \eta}{R} g \varphi_{\lambda}
$$

where $\eta$ is the quantum efficiency of the detector and $g$ is the geometrical factor which depends on the pixel size, the slit width, the possible pixel binning, etc. (see Picat et al. 1994, for more details). $\varphi_{\lambda}$ is the spectral flux at the entrance of the telescope. After sky subtraction, the signal of the object on the detector will be:

$$
S=\left[\Phi_{O}+\left(\Phi_{S}-\Phi_{S^{\prime}}\right)\right] T
$$

The sky residuals are considered as sources of noise which can be written

$$
N=\sqrt{\sigma_{C C D}^{2}+\sigma_{\text {photons }}^{2}}+\mu_{\text {sky }}+\mu_{\text {flat }}+\mu_{\text {lines }}
$$

where the first two terms are the standard sources of noise (readout and photon noises) which add quadratically. The other ones are additive because they are systematic and occur always on the same pixels.

In the perfect case, only the photon noise has to be taken into account provided we neglect the readout noise of the detector (this will be assumed throughout the rest of this paper). This noise term can be written as $\sigma_{\text {photons }}^{2}=(2) \Phi_{S} T$ with a factor (2) depending how the sky is subtracted (pixel-to-pixel or average of a large number of sky rows). The $\mathrm{S} / \mathrm{N}$ per pixel is therefore:

$$
(S / N)_{\text {perfect }}=\frac{\Phi_{O} T}{\sqrt{(2) \Phi_{S} T}} \propto \frac{\Phi_{O}}{\Phi_{S}} \sqrt{\frac{D^{2} \eta}{R} g T}
$$

as mentioned at the beginning of the paper. But in the "real world", one has to explicit the additive terms of the noise estimate:

$\mu_{\text {sky }} \sim \beta \Phi_{S} \mathrm{~T}$ represents the temporal and/or the spatial variations of the sky below the object. 
$\mu_{\text {flat }} \sim \epsilon \Phi_{S} T$ corresponds to the flatfield errors remaining after the sky subtraction.

$\mu_{\text {lines }} \sim \omega \Phi_{\mathrm{S}} \mathrm{T}$ is due to sampling errors, grating misalignment or slit width variations, mostly sensitive in the spectral range dominated by the strong sky emission lines.

All these factors are simply proportional to the sky flux and the exposure time, if we assume that the object is faint enough to be negligible with respect to the sky. In that case, the $\mathrm{S} / \mathrm{N}$ will be:

$$
(S / N)_{\text {real }}=\frac{\Phi_{O} T}{\sqrt{(2) \Phi_{S} T}+(\beta+\varepsilon+\omega) \Phi_{S} T}
$$

At high flux level (or long integration time), it will reach a limit

$$
(S / N)_{l i m i t} \sim \frac{\Phi_{O}}{\Phi_{S}} \frac{1}{\beta+\varepsilon+\omega}
$$

which does not depend anymore on the diameter of the telescope $D$, the resolution of the instrument $\mathrm{R}$ nor the integration time $\mathrm{T}$. Increasing the diameter of the telescope or the total exposure time, with everything else kept constant, will not allow to reach fainter limiting magnitudes, but the limiting $\mathrm{S} / \mathrm{N}$ will be reached faster!

\section{WHAT IMPROVEMENTS FOR THE LONG SLIT SPECTROSCOPIC MODE?}

This concern is not new, and some improvements can be expected mainly from the high quality of the optical design of the instruments. This means a reduction of the optical distortions and mechanical flexures, and a careful inspection of the slit quality and its alignment. In the long slit spectroscopic mode, the sky and the object signals are integrated at the same time, but not at the same position on the detector. The temporal sky fluctuations which are known to be important for long exposure times are then minimized. Moreover, with two-D detectors the underlying sky can be interpolated from the sky spectrum on each side of the object to minimize the spatial variations of the sky spectrum. But in any case flatfield and sky lines residuals will dominate the noise structure for very faint objects. In this mode the $\mathrm{S} / \mathrm{N}$ will be limited to:

$$
(S / N)_{\text {limit }} \sim \frac{\Phi_{O}}{\Phi_{S}} \frac{1}{\varepsilon+\omega}
$$

and the "saturation" time (the integration time necessary to reach $1 / 2$ of the limiting $S / N$ ) can be expressed as:

$$
T_{s a t} \sim \frac{1}{\Phi_{S}(\varepsilon+\omega)^{2}}
$$

As a numerical example, one can compare two similar spectrographs in use at ESO, namely EFOSC on the 3.6-m telescope and EMMI on the NTT, in their low resolution mode $(\mathrm{R}=$ 400). In the spectral region around $5000 \AA$ where the sky spectrum is dominated by the continuum, the saturation time of EFOSC is about five hours for a object-to-sky ratio of 0.1 and the $\mathrm{S} / \mathrm{N}$ limit is about ten per pixel of 0.675 size, while for EMMI it corresponds to 28 
hours for the same object but with a pixel size of 0."44. In the red part of the spectrum around $8000 \AA$ where the sky spectrum is dominated by emission lines of atmospheric molecular bands, the saturation time of EFOSC is shorter than 30 minutes, while for EMMI it is of about two hours, both for an $\mathrm{S} / \mathrm{N}$ limit of two per pixel. There are two major differences between the two spectrographs which can explain these numbers: first, the CCD in use on EFOSC is a thinned Tek CCD with a higher quantum efficiency than the thick coated Thomson CCD EMMI (it has changed now.). Second, the pixel sizes of the two spectrographs are quite different and the spatial and spectral sampling are quite better in the second case. Undersampling and high efficiency are consequently the two major points which favor an easy detection of the limiting effects in low resolution spectroscopy. One can also note that a VLT (D = eight-m) with a pixel size of 0.3 and a blue thinned CCD will be equivalent to EFOSC in the estimate of the saturation time!

Some alternatives such as beam switching mode have already been proposed to solve this problem and increase the sensitivity of the spectrographs. It allows the removal of the spatial errors, as the sky and the objects are recorded through the same optical path (or the same fiber). But as they are not anymore recorded at the same time, the temporal fluctuations of the sky brightness can be large for long exposure times and they are difficult to monitor accurately.

Another solution may come from a technique similar to the "shift-and-add" one introduced by Tyson (1988) for very deep imaging. Individual exposures are recorded with random spatial shifts and the spatial defects on the detector do not correspond to the same position with respect to the objects and can be removed by median filtering. In spectroscopic mode, this has been attempted in a few cases (Cowie and Lilly 1989), but it requires a long individual exposure time to reach the photon-noise regime even with a low readout noise $C C D$, and the shifts are not easy to produce inside the slit. Moreover, to obtain a significant gain in the $\mathrm{S} / \mathrm{N}$, the number of shifts must be large (typically larger then ten), and this is hardly compatible with the previous requirements of a long enough individual integration time. So this method can be efficient only in a very few specific cases!

\section{PRINCIPLE OF THE "VA-ET-VIENT" MODE}

Because of these remaining $\mathrm{S} / \mathrm{N}$ limitations, we propose to implement a new mode of observation that we call "Va-et-Vient". It is like a beam switching method but instead of reading the CCD at the end of each elementary exposure, the charges are shifted as described in Fig. 1. After each exposure, the telescope is moved from the object to a sky reference so that the object and the sky are integrated through the same pixels and recorded on two different zones on the CCD. The shifting period can be chosen as small as necessary to freeze out the temporal sky fluctuations, a typical value being three to five minutes. Finally, there is only one readout at the end of the sequence. Note that this idea of chopping technique was already used in the 70's in spectroscopy with photon counting detectors (Shectman and Hiltner 1976), and tentatively implemented in an imaging polarimeter (McLean et al. 1981).

In the "Va-et-Vient" spectroscopic mode, the sky subtraction must be done pixel by pixel and the photon noise in the remaining object spectrum is twice that of the sky flux, instead of only once in the case of a long slit with a sky modeling:

$$
\sigma_{\text {photons }}^{2}=2 \Phi_{S} T
$$



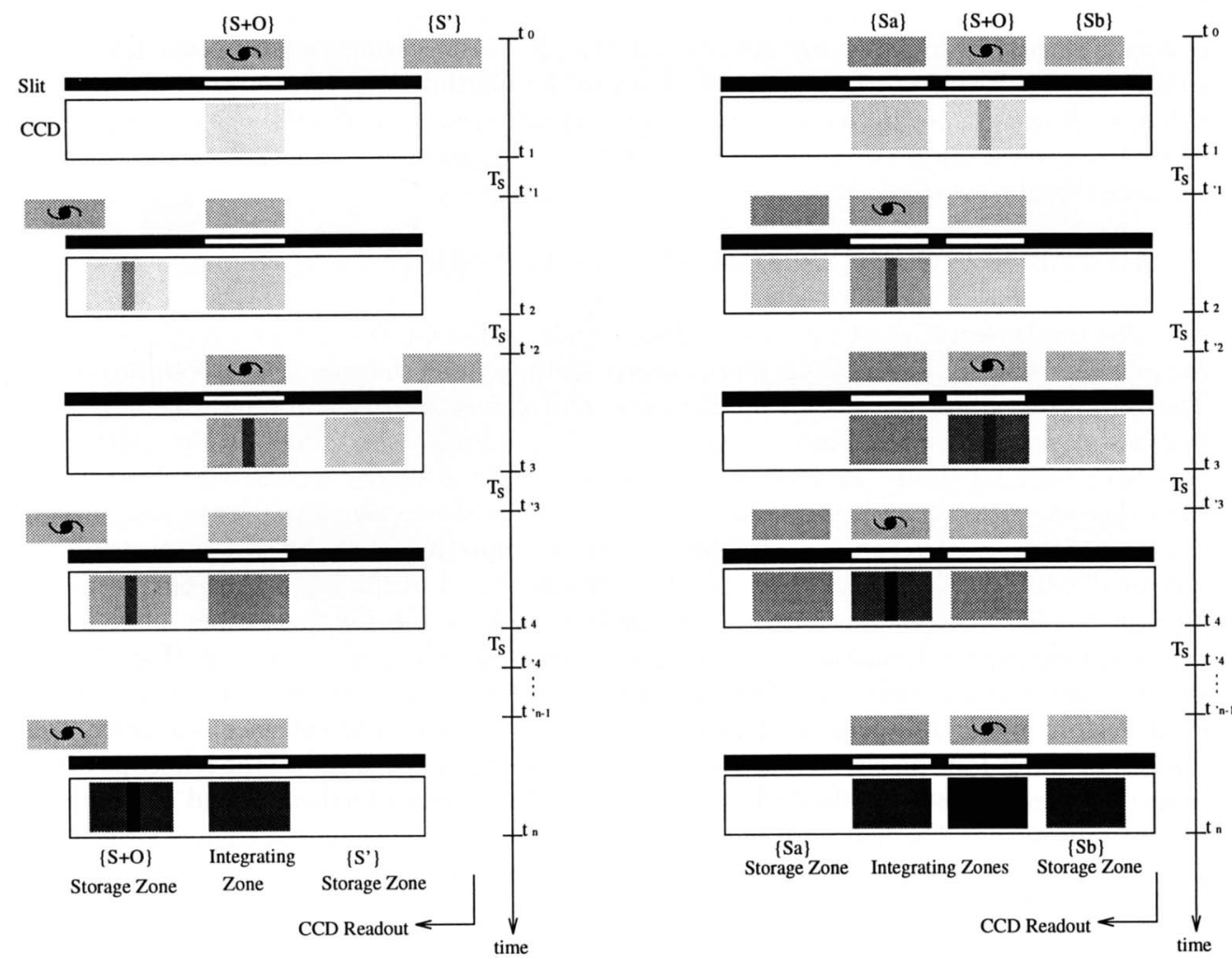

Fig. 1. Configuration of the CCD and the telescope during a "Va-et-Vient" spectroscopic exposure. Relative positions of the telescope pointed on the object and on the adjacent sky appear on the figure as the corresponding charges are moved on the CCD. The projection of the slit on the CCD determines the physical integration zones. In order to avoid any mixing of the data, a slitlet is needed to mask the light from the sky in the storage zones. Only $50 \%$ of the total integration time is spent on the object, while the other $50 \%$ is spent on the sky. (right) Same as before but in order to optimize the telescope time, two slitlets are used and the shifts of the pixels on the CCD are related spatially with the shifts of the telescope. In that case, the object in integrated $50 \%$ of the time in one slit and $50 \%$ in the other slit, both in the same strip on the CCD, while two strips of sky spectrum are recorded on the CCD. In the final image, three strips are produced for one object spectrum (from Cuillandre et al. 1994). 
A major advantage of the method is that the flatfielding residuals apply only on the object flux, and there is no more sky lines sampling errors (the sky is nearly perfectly subtracted). The $\mathrm{S} / \mathrm{N}$ is therefore:

$$
S / N=\frac{\Phi_{O} T}{\sqrt{\sigma_{C C D}^{2}+2 \Phi_{S} T}+\beta \Phi_{S} T+\varepsilon \Phi_{O} T}
$$

It is mainly limited by temporal sky fluctuations $(\beta)$. For an elementary exposure time short enough, "Va-et-Vient" spectroscopy will work in the photon noise regime, even at high flux when working with long integration time $\mathrm{T}$, large telescope and small resolution. To test more quantitatively the ideas developed above, we have implemented a laboratory experiment to simulate the observing conditions in faint object spectroscopy.

\section{THE LABORATORY EXPERIMENT AND ITS RESULTS}

The implementation of the "Va-et-Vient" mode on the CCD is rather straightforward as the physical structure of the CCD allows charge shifting along the columns in both directions. The parameters which must be included in the controller software are: the shifting period, the number of periods for one exposure and the number of lines to be shifted. In our experiment we used a backside illuminated 512 × 512 Thomson CCD driven by a controller developed at ESO. The sky spectrum was simulated by a combination of two lamps, a halogen lamp for the sky continuum and a Neon spectral lamp for the emission lines. For the galaxy spectrum, we also used a halogen lamp, but with a blue filter introduced in the beam. The last beam was directly focussed on the entrance of the spectrograph, while the two other beams were combined through a beam splitter, giving a uniform illumination of the slit (Fig. 2). The spectrograph was made of two conjugated lenses with a low dispersion grism in the parallel beam region, giving a dispersion of $25 \AA$ /pixel on the detector. The entrance is a slitlet, with a width of about four pixels, so that the resolution was $100 \AA$. To simulate the telescope offsets from the object to the sky, the "galaxy" lamp was alternatively switched on and off, with the sky one always on. The lamp fluxes and the exposure times were adjusted to give a signal per pixel equivalent a one-hour exposure on a 4-m telescope. The details of the experiment can be found in Cuillandre et al. (1994).

The first results were aimed at showing if there was a degradation of the output signal in "Va-et-Vient" mode. More quantitatively, we measured an additive noise lower than one electron rms, and no degradation of the MTF (60 shifts of 100 lines) as we used a high quality CCD with a very small number of traps. Charge Transfer Efficiency (CTE) losses were only detectable for more than 1000 shifts of 100 lines. A by-product of this method is a measure the CTE with a value quite similar to the one measured with other methods.

The data reduction procedure for the spectra was nearly the same as in long slit spectroscopy, except a few changes in the sequence of operations (see table below). In particular, the sky subtraction in "Va-et-Vient" mode must be done pixel-by-pixel before any other process to keep exactly the same sampling between the object and the sky.

The experimental results on long slit and "Va-et-Vient" methods are displayed in Fig. 3. They clearly show the saturation of the $\mathrm{S} / \mathrm{N}$ with long slit spectroscopy, while the $\mathrm{S} / \mathrm{N}$ still increases with flux in the "Va-et-Vient" mode. Both sets of data were obtained in the same 


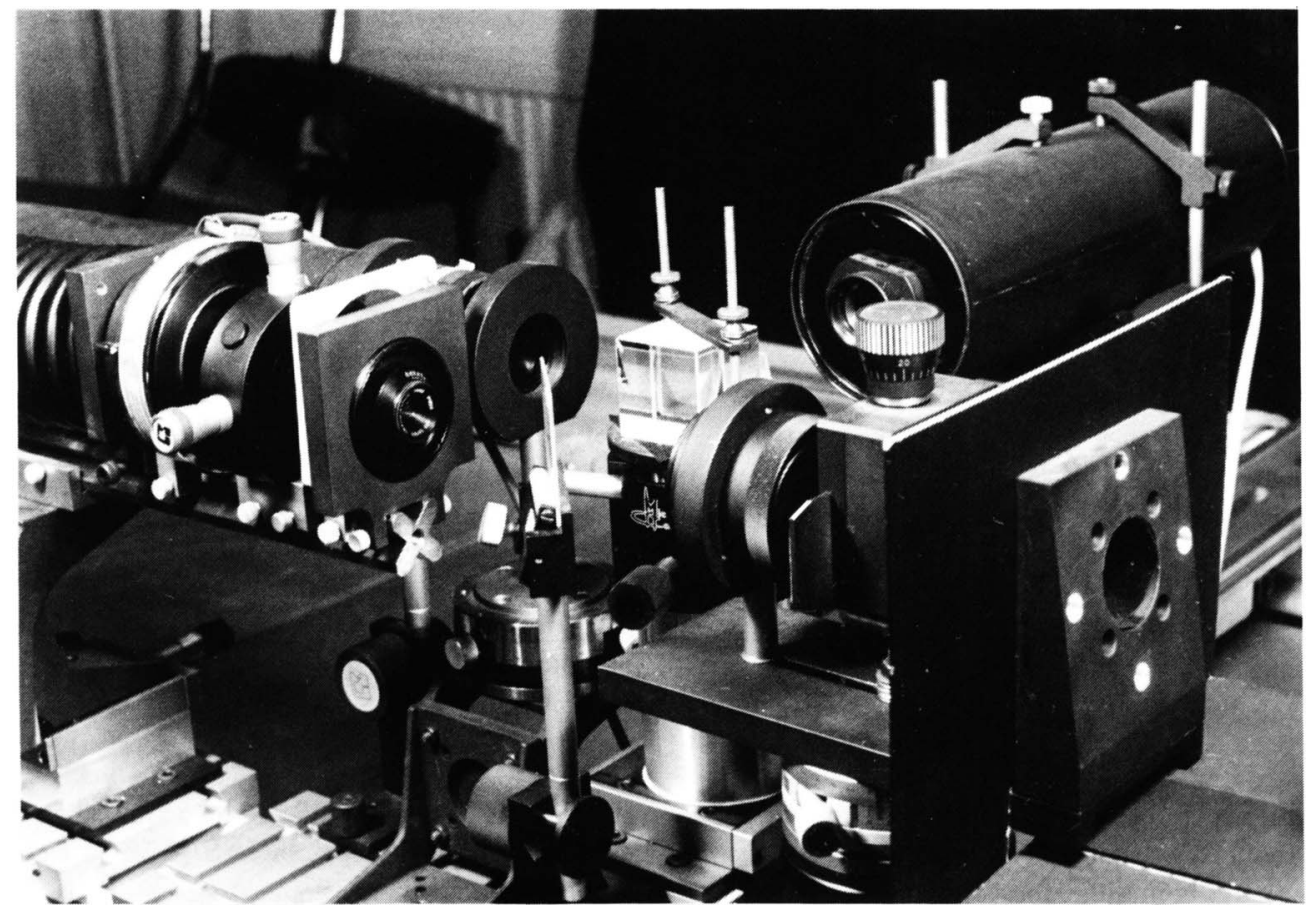

Fig. 2. Picture of the optical bench used in the experiment.

\section{TABLE 1}

Major steps in the data reduction procedure, both in long slit and in "Va-et-Vient" spectroscopic modes.

\begin{tabular}{ll}
\hline \hline Long slit & "Va-et-Vient" \\
\hline $\begin{array}{l}\text { bias subtraction } \\
\text { flatfielding }\end{array}$ & $\begin{array}{l}\text { bias subtraction } \\
\text { sky subtraction } \\
\text { (pixel-by-pixel) }\end{array}$ \\
$\begin{array}{l}\text { sky subtraction } \\
\text { flatielding } \\
\text { object extraction }\end{array}$ & object extraction \\
wavelength calibration & wavelength calibration
\end{tabular}




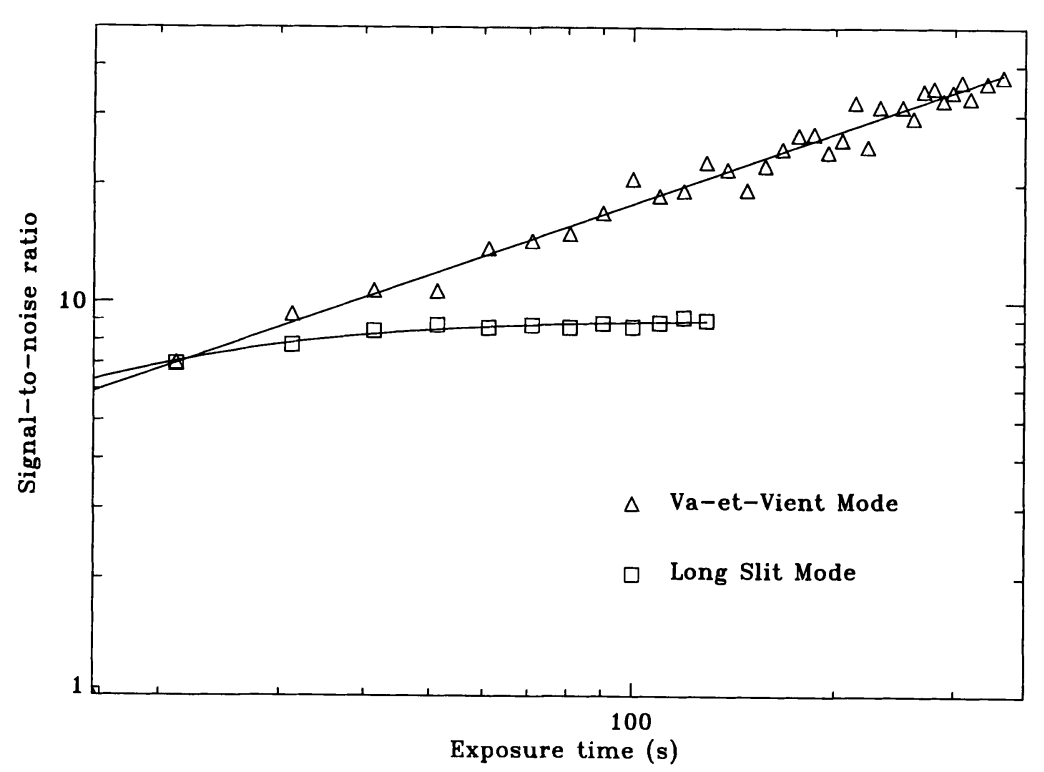

Fig. 3. S/N versus integration time for the long slit and the "Va-et-Vient" modes acquired and reduced in the same conditions, from the laboratory experiment. The gain in S/N in "Va-etVient" is mainly due to the better subtraction of the sky emission lines.

experimental conditions, with an object-to-sky ratio of 0.2 approximately. The gain in magnitude can reach 0.6 magnitudes in the continuum for an object-to-sky ratio of 0.1 , and can be as large as 2.5 magnitudes in spectral regions dominated by strong emission

\section{TELESCOPE IMPLEMENTATION AND EXPECTED PERFORMANCES}

Preliminary tests on a telescope were successfully attempted on the ESO NTT. We chose an elementary exposure time of three minutes and the dead time (shift of the telescope and shift of the charges) between them was less than five seconds. Moreover, to save time during the shifts, we kept the telescope guiding on the object position while it was tracking on the sky because of the long recentering time on the guide star. As no link was implemented between the CCD controller and the Telescope Control Software (TCS), we worked in a semi-automatic way, the shifts of the telescope and the starting of individual exposures being manually controlled.

Because we did not have enough time allocated on the experiment with the spectrograph available on the NTT, the tests of the "Va-et-Vient" capabilities were not obtained in the optimal conditions discussed above. In particular they were performed in a too high dispersion mode (typically $5 \AA /$ pixel) to be the most efficient, compared to standard spectroscopy. The total integration time was then not sufficient to reach the limiting regime in the long slit mode. However we found that the sky subtraction of strong emission lines was significantly improved. 
The next step in the implementation of the "Va-et-Vient" will be to use a fully automatic procedure with a link between the TCS and the CCD controller at the Canada-France-Hawaii Telescope in the Pegasus software environment, following the observing sequence detailed below (the steps written in italics are repeated $n$ times, where $n$ is the number of sequences in the total integration):

- Initialize the CCD

(Telescope is guiding)

- Open the shutter

- Individual exposure ( $t$ )

- Close the shutter

- Move the charges on the CCD

- Move the telescope

(Telescope is tracking)

- Open the shutter

- Individual exposure ( $t$ )

- Close the shutter

- Move back the charges

- Move back the telescope

\section{- Read the CCD}

The new parameters which have to be defined in the user interface are: the number of "Vaet-Vient" $\mathrm{n}$, the individual integration time $t$, the number of lines to shift on the CCD $\Delta Y$, and the shift of the telescope $\Delta \alpha, \Delta \delta$. In these conditions, the total integration time will be $T=2 n t$. Moreover, in "Va-et-Vient" mode with one slit, $\Delta Y$ will be independent of $\Delta \alpha, \Delta \delta$, otherwise they will be combined.

\section{CONCLUSION}

In conclusion, we have demonstrated numerous interests of the "Va-et-Vient" mode for faint object spectroscopy and many others will be explored in a near future. In particular, it has long been known that the flatfield corrections with thinned CCDs are quite difficult to apply in spectroscopy because of the strong fringes which appear in the red part of the spectra. They are generally badly removed, especially when the sky is spatially and spectrally varying on the chip. In a "Va-et-Vient" mode, they will apply on the object flux only, as we checked it in the laboratory and their contribution to the underlying sky will disappear in the sky subtraction.

Another potential interest of the "Va-et-Vient" mode will be the possibility to draw curved slits, with a much better efficiency in the data reduction as the spectral sampling residuals are removed. This was already used by Soucail et al. (1988) for acquiring the spectrum of the giant arc in Abell 370, with a MOS starplate punched with adjacent holes which followed the shape of the arc itself. The data reduction and the sky subtraction were not easy to calibrate accurately, because the transmission and the sampling were not constant and not well defined along the slit. In a "Va-et-Vient" mode, the object and the sky would be obtained through the same slit, avoiding the duplication of these very special apertures! 
Finally, from the laboratory experiment, we predict a gain of one to two magnitudes in the limiting magnitude for low resolution spectroscopy (Picat et al. 1994). This could be a major gain for the next generation of very large telescopes. From our point of view, the implementation of the "Va-et-Vient" on future faint object spectrographs will be unavoidable. Even if the observing procedures are slightly more complicated, the data reduction is straightforward and does not differ very much from standard procedures. "Va-et-Vient" can be extrapolated on different purposes such as Multi-Object Spectroscopy. A double slit is used for each selected object, but the same shift between each object and a corresponding sky is required all over the field, which can be difficult to maximize the number of objects. A dedicated software will consequently have to be developed for the selection of objects in this configuration. Moreover, with the double slit system, three spectroscopic strips per object will be acquired on the CCD and the number of objects per mask will be limited to 20 typically for a ten by ten arcminute field of view. But this will be a solution to keep the efficiency of two-D detectors on the spectroscopic observations of very faint objects.

\section{REFERENCES}

Boksenberg, A. 1975 in Image Processing Techniques in Astronomy, C. de Jager and H. Nieuwenhuizen, eds., Reidel, Dordrecht, p. 59

Colless, M. M., Ellis, R. S., Taylor, K. and Hook, R. N. 1990 MNRAS 244, 408

Colless, M. M., Ellis, R. S., Broadhurst, T. J., Taylor, K. and Peterson, B. A. 1993 MNRAS 261, 19

Cowie, L. L. and Lilly, S. J. 1989 ApJ 336, L41

Cowie, L. L., Gardner, J., Wainscoat, R. J. and Hoddap, K. W. 1994, preprint

Cuillandre, J. C., Fort, B., Picat, J. P., Soucail, G., Altieri, B., Beigbeder, F., Dupin, J. P., Pourthié T. and Ratier, G. 1994 A\&A 281, 603

Lilly, S. J., Cowie, L. L. and Gardner, J. P. 1991 ApJ 369, 79

McLean, I. S., Cormack, W. A., Herd, J. T. and Aspin, C. 1981 Proc. SPIE 290, p. 155

Picat, J. P., Cuillandre, J. C., Fort, B. and Soucail, G. 1994 Proc. SPIE 2198, Astronomical Telescopes and Instrumentation for the 21st Century, D. L. Crawford, ed., p. 1274

Shectman, S. A. and Hiltner, W. 1976 PASP 88, 960

Soucail, G., Mellier, Y., Fort, B., Cailloux, M. and Mathez, G. 1988 A\&A 191, L19

Tresse, L., Hammer, F., Le Fèvre, O. and Proust, D. 1993 A\&A 277, 53

Tyson, J. A. 1988 AJ 96, 1

\section{DISCUSSION}

TINBERGEN: This is an established technique in polarimetry. Also a low-contrast high-signal situation. Stenflo's group in Zurich has an operational solar spectropolarimeter using this technique. You may like to compare notes with them to see how far you can push this technique for the VLT.

SOUCAIL: Yes, we knew that similar techniques were used in polarimetry for low contrast detections. In the case of the Sun, the problems may be different as it is not a question of low flux but low contrast. For faint object spectroscopy, we have to combine the effects of undersampling with those of low spectral flux. 
FINGER: Why does this method only work at very low resolution?

SOUCAIL: "Va-et-Vent" spectroscopy is less efficient than standard long-slit spectroscopy while the defects of the spectrograph are, in some sense, negligible (flatfielding residuals, undersampling or poor sky subtraction), which is the case when the signal is split in many spectral elements. But at low resolution they become dominant and limit the $\mathrm{S} / \mathrm{N}$ of the data for long integration times.

LEACH: This method has been applied to stellar polarimetry with TI 800 x 800 CCDs with CTE not being a serious problem for polarimetry accuracy down to $10^{-3}$. Do you see streaks due to charge trapping?

SOUCAIL: We have tried to work in regions of the CCD where no defects were apparent as far as possible and did not detect any strong streaks. In any case, we can limit the number of shifts to a few tens, with a few tens of lines for each shift, which minimizes the sensitivity of the method to CTE limitations.

CULLUM: In your two slit method, would it not be better to record the two object fields separately on the CCD like for the sky? (ie. use four zones on the CCD per object, two sky and two object.) By adding the charges recorded by two separate slits (and recorded by different pixels) one would expect worse subtractor of the sky.

SOUCAIL: This is not exactly the case, because in the two slit mode, you have three strips of data at the end of the exposure, the central one corresponding to the sum of the charges integrated through the two slits for the (object + sky). On the adjacent strips, you have the sky charges recorded through each slit and by simply adding them, you recover the correct signal to subtract to the central strip. Indeed this would be perfectly correct if there was no flatfield correction to apply, otherwise the correction on the object signal is not perfectly applied. To avoid this problem, the solution proposed with four strips is better, but it also takes more space on the CCD.

TINBERGEN It is suitable for all low contrast situations. The basic thing is you avoid flat fielding by using the same pixel for measuring both large quantities, whose small difference you are interested in. 